\title{
ASO Author Reflections: Disparities in Outcome and Surgical Management Following Neoadjuvant Chemotherapy for Breast Cancer
}

\author{
Theresa Relation, MD, PhD $\mathbb{D}$, and Bridget A. Oppong, MD \\ Division of Surgical Oncology, Department of Surgery, The Ohio State University, Columbus, OH
}

\section{PAST}

While breast cancer is the most commonly diagnosed cancer among women, the burden of disease disproportionately falls on Black women, who are more frequently diagnosed at advanced stages and have higher mortality rates. Previous studies have identified socioeconomic barriers to treatment and diagnosis, higher rates of aggressive tumor phenotypes, and earlier age of onset as factors contributing to this observed disparity. ${ }^{1}$ While neoadjuvant chemotherapy (NAC) has proven useful in reducing overall morbidity and mortality among breast cancer patients, White women are more likely to complete NAC treatment, and Black women are more likely to experience nodal recurrence after NAC. ${ }^{2}$ Combined, these observed patterns demonstrate a significant and concerning outcome disparity between non-Hispanic White and Black patients.

\section{PRESENT}

Our analysis revealed a continuing pattern of adverse socioeconomic variables in Black women, including lower income, educational attainment, and rates of insurance coverage. In examining tumor and clinical features, Black women showed a higher proportion of advanced disease, more poorly differentiated tumors, lower response rates to chemotherapy, and lower likelihood of receiving immunotherapy. White women were more likely to receive

(C) Society of Surgical Oncology 2021

First Received: 22 January 2021

Accepted: 22 January 2021;

Published Online: 3 March 2021

B. A. Oppong, MD

e-mail: bridget.oppong@osumc.edu breast-conserving surgery and sentinel lymph node biopsy versus axillary lymph node dissection (ALND). ${ }^{3}$ The disproportionate use of ALND in Black women is a disconcerting finding considering the higher complication rates, especially lymphedema.

\section{FUTURE}

The devastating impact of COVID-19 on minority communities has forced us to confront health disparities across many diseases, including breast cancer. ${ }^{4}$ There remains an urgent need to investigate differences in outcome and understand the factors contributing to disparities. Future interventions must be directed by qualitative studies focused on elucidation of patient preferences, cultural beliefs, and surgeon practice patterns to address disparate treatment. A challenge for healthcare providers is to analyze our own clinical decision-making to recognize the unconscious biases that lead to inequity.

FUNDING The National Cancer Database (NCDB) is a joint project of the Commission on Cancer $(\mathrm{CoC})$ of the American College of Surgeons and the American Cancer Society. The CoC's NCDB and the hospitals participating in the CoC's NCDB are the source of the deidentified data referenced herein; they have not verified and are not responsible for the statistical validity of the data analysis or the conclusions derived by the authors.

DISCLOSURE The authors declare that they have no conflicts of interest.

\section{REFERENCES}

1. Williams DR, Mohammed SA, Shields AE. Understanding and effectively addressing breast cancer in African American women: unpacking the social context. Cancer. 2016;122(14):2138-49. h ttps://doi.org/10.1002/cncr.29935. 
2. Killelea BK, Yang VQ, Wang S-Y, et al. Racial differences in the use and outcome of neoadjuvant chemotherapy for breast cancer: results from the National Cancer Data Base. J Clin Oncol. 2015;33(36):4267-76. https://doi.org/10.1200/JCO.2015.63.7801.

3. Relation T, Obeng-Gyasi S, Bhattacharyya $\mathrm{O}$, et al. Racial differences in response to neoadjuvant chemotherapy: impact on breast and axillary surgical management. Ann Surg Oncol. 2021. h ttps://doi.org/10.1245/s10434-021-09657-w.
4. Obeng-Gyasi S, Oppong B, Paskett ED, Lustberg M. Purposeful surgical delay and the coronavirus pandemic: how will black breast cancer patients fare? Breast Cancer Res Treat. 2020;182(3):527-30. https://doi.org/10.1007/s10549-020-05740-0.

Publisher's Note Springer Nature remains neutral with regard to jurisdictional claims in published maps and institutional affiliations. 